\title{
Electronic Voting Machine Using a Microcontroller
}

\author{
Payal Yadav ${ }^{1}$, Ansh Gupta ${ }^{2}$ \\ ${ }^{1,2}$ Department of Electrical Engineering, \\ Delhi Technological University, Delhi, India \\ Email:payaa27@gmail.com,gupta.ansh256@gmail.com
}

\begin{abstract}
Electronic voting machine is an efficient and fast solution that allows people to choose their representative in a systematic manner. It is a simple device that has replaced the conventional ballot papers used to record votes. Also, in large populations, the ballot system failed, whereas EVMs proved to be successful and reliable. This project describes a real-time voting machine that is designed using the microcontroller AT89C51 and the software program used is written in assembly language. It is a real-time operating device that produces lesser errors and is easy to operate. Once the votes are cast, the result can be produced in no time just by pressing the result button.
\end{abstract}

Index Terms - Electronic Voting Machine, Liquid Crystal Display, Light Emitting Delettevice, Microcontroller.

\section{INTRODUCTION}

India is the-largest democratic-country with a total of 850 million recorded voters. The election commission has taken the creative project of recording, storing and counting votes across the nation transparently and securely that is backed by proper legal support. The Election commission has been successfully using EVMs for performing elections for the last 23 years.

The electronic Voting Machine maintains all the traditional ballot paper voting system characteristics while making voting a lot more expedient. It also saves a lot of time and manpower being fast and absolutely reliable.

In addition to this, EVM helps maintain the voting secrecy and the result is $100 \%$ tamperproof. EVMs have been of great use to India since they were first introduced in 1982 and subsequently became very popular as well. It is highly user friendly as it provides the result with just a button and also, the voters have only to press one button to cast their vote. The AT89C51 microcontroller used is a high performance, low power 8bit microcomputer with $4 \mathrm{~K}$ Bytes of programmable flash memory.

EEPROM memory is used to store the voting sum. The advantage of this memory is that it keeps data in memory even after power is turned off. The microcontroller provides the following features: 128 bytes RAM, -32 I/O lines, two 16-bit counters/timers, fullduplex UART serial channel and an on-chip oscillator and clock circuit. The Idle Mode halts the CPU while allowing the RAM, serial port, timers, and interrupt system to continue operating.

\section{OUTLINE OF E-VOTING TECHNOLOGIES}

Document Ballot Voting System - This system was used to keep a count of all the votes marked on a ballot sheet electronically. Direct Recording Electronic voting system - In this system, ballot display was provided using a touch display or an electro-optical component. Public NetworkkDRE Voting System This system uses an electronic ballot to record the entire data throughout the voting process and sends it through a public network to a central location. The Indian EVMs - These are designed and developed by two manufacturing units that are owned by the government, i.e. Bharat Electronics Limited (BEL) and Electronics Corporation of India (ECIL).

The Indian EVMs - These are designed and developed by two manufacturing units that are owned by the government, i.e. Bharat Electronics Limited (BEL) and Electronics Corporation of India (ECIL).

Figure 1. Components of an EVM

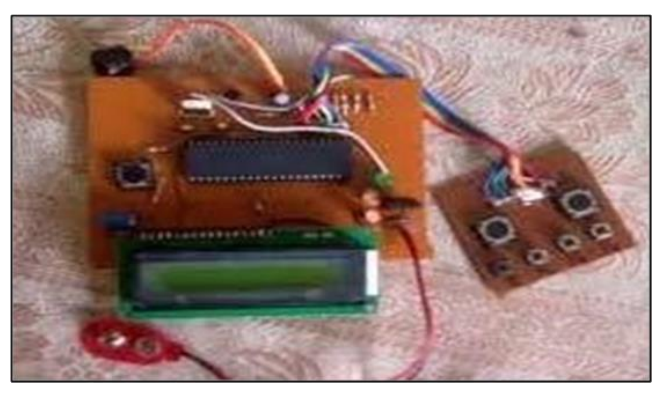




\section{BLOCK DIAGRAM OF EVM}

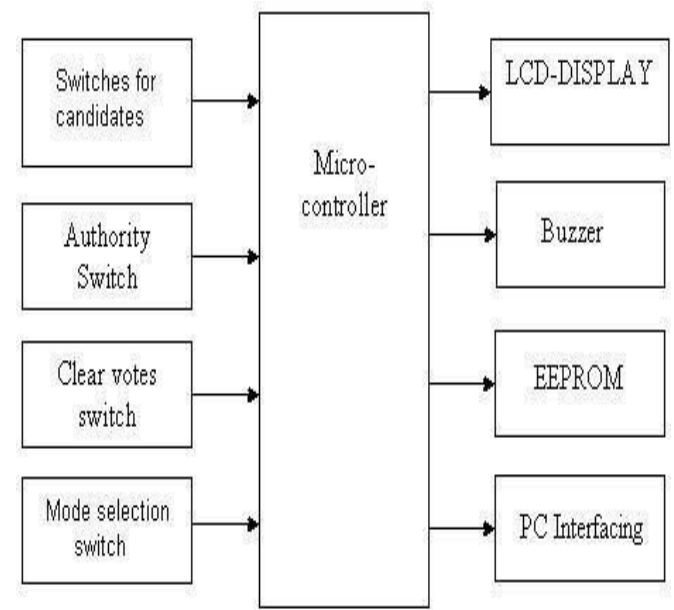

Figure 2. Block Diagram of EVM

\section{BLOCK DESCRIPTION}

1. Microcontroller- Based on the signal received from the switches, the mode of operation is decided. Its job is to increment the data when the switches are pressed against the name of each candidate in the voting model. Also in counting mode, it fetches the data from memory and sends it to the display devices.

Figure 3. Microcontroller internal structure

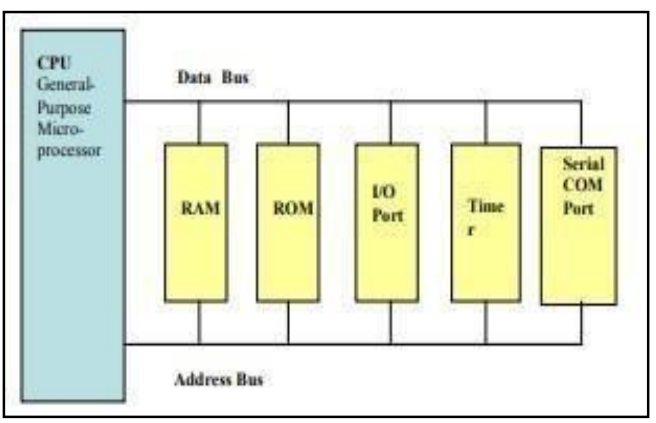

2. LCD - An alphanumeric display is used instead of a seven-segment display because it can display both alphabets and numbers and special symbols that make LCD more user-friendly.

3. LED - Light-emitting diodes are semiconductor devices made out of silicon. These LEDs emit photons when current passes through them, which makes them appropriate for indicator lamps.

4. CONTROL SWITCHES - These are - clear votes, a controller switch, and total votes.

\section{CIRCUIT DIAGRAM}

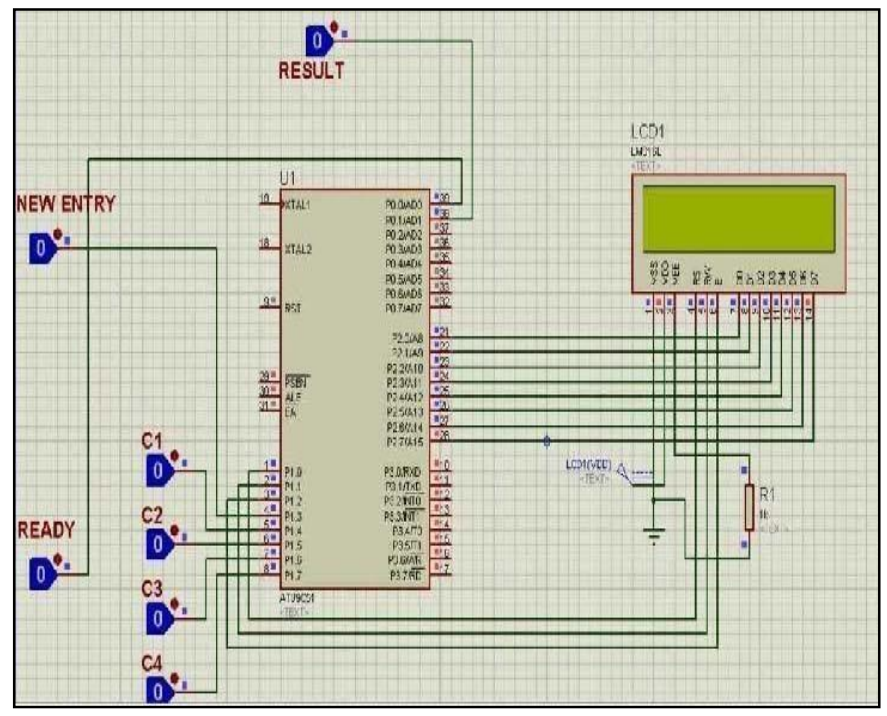

FIGURE 4. PROTEUS MODEL OF THE EVM

\section{WORKING OF EVM}

Power on- The supply when turned 'ON' is indicated by a red light.Mode Selection- It has two modes, namely, voting mode, i.e., when the toggle switch is on VCC. In this mode, the message "Please Vote" is displayed on the screen. After a vote is cast, the controller switch is pressed by the voting authority and the next mode is Counting Mode, i.e. when the toggle is on GROUND. In this mode, once the respective key is pressed, each candidate's total number of votes is displayed on the screen.

1. Clear Mode - This switch ensures that all of the preceding data has been cleared and the EVM is ready for another election as it clears all the entries. Usually, this switch is pressed before the voting procedure begins.

2. Buzzer Signal - Pressing of a key when a vote is a buzzer soundController Switch specifies cast - This switch is always under the control of the voting authority as it is provided for enabling the keypad in the voting mode to let a voter cast his vote. 


\section{MAIN UNITS OF EVM}

1. Control Unit - The most important part of an EVM is a control unit consisting of a power supply, switch, LCD, microcontroller, and result key. This unit is used to keep track of the input buttons. It increments the content of the memory locations reserved for each candidate whenever a-key is pressed.

Once a vote is a cast, it disables the input switches for a calculated interval of time to avoid tampering in the form of multiple ballots cast by one voter. Results can also be displayed using the 'Result' switch.

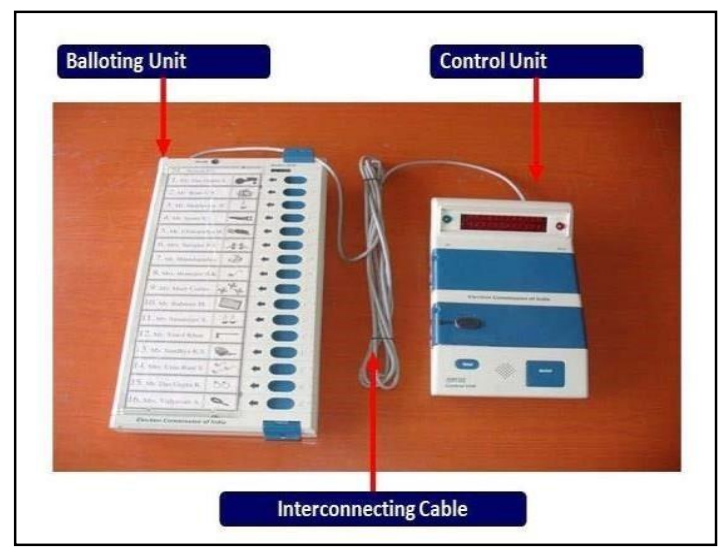

Figure 5. Control Unit and Ballot Unit

2. Ballot Unit - It is the ballot box that displays the list of all the candidates. It works as a facility to display various party names and symbols. A single Ballot Unit can contain up to 16 candidates and therefore, by connecting 4 ballot units, a total of 64-candidates can be accommodated in a single election.

Also, it is user-friendly and demands no training for the same.

\section{ISSUES OF EVM}

Electoral officials are examining around the world and trying to address various challenges such as system, and ease of preservation of the system along with an errorfree operation and tamperproof results acceptability by common people in remote areas, system operation as close to the conventional ballot.

1. Bias - Many times, the candidates feel that the order in which their names are displayed on the EVM affects their vote counts because they believe that terms displayed first will be preferred more.

2. Verifiability - Earlier, before introducing the EVMs people, were confident that their votes were secured. The votes cast on paper or on punch cards were held accountable until the introduction of EVMs, after which the government had no oversight into the production of such systems handled by private companies beyond the choice of using them or not.

Candidates can know how many people have voted for them from any polling station, which results in misconduct with the voters due to lesser votes in any area. That is why EVMs are now created to display only the overall total results from an assembly or Sabha.

\section{NEED FOR FURTHER DEVELOPMENT}

Even though the EVMs are designed for the electoral system of a country, it needs some alterations. The authentication of voters has to be stretched to another level, i.e. beyond the VOTER ID. Either the fingerprint could be scanned or the face detection system could be used to avoid multiple votes by a single voter.

Since now the EVMs are equipped with networking capabilities allowing voters to vote from anywhere in the world, there must be a more secure system and also, those votes must be brought in as soon as possible so that the result is not delayed.

\section{FUTURE SCOPE}

As we know that currently the total number of votes that can be catered by an EVM is 3840 . So, to be able to provide EVMs to a larger audience in a single day such that the total duration of an electoral process is reduced, the memory and internal architecture also need to be altered. The main focus now is to increase the internal memory size of an electronic voting machine. The idea is to now incorporate some other method for authentic voting, such as iris detection or fingerprint scanner.

\section{RESULTS}

The voting machine can be moderated as per the system and number of candidates. Also, post-2013 the M3 EVMs can serve a maximum of 384 candidates by connecting 24 ballot units together at a time. The control switches help in an easy and fast calculation of results and provide complete information about the total votes cast, displayed on the LCD screen. The clear button allows the machine to be used multiple times, thereby reducing costs. 


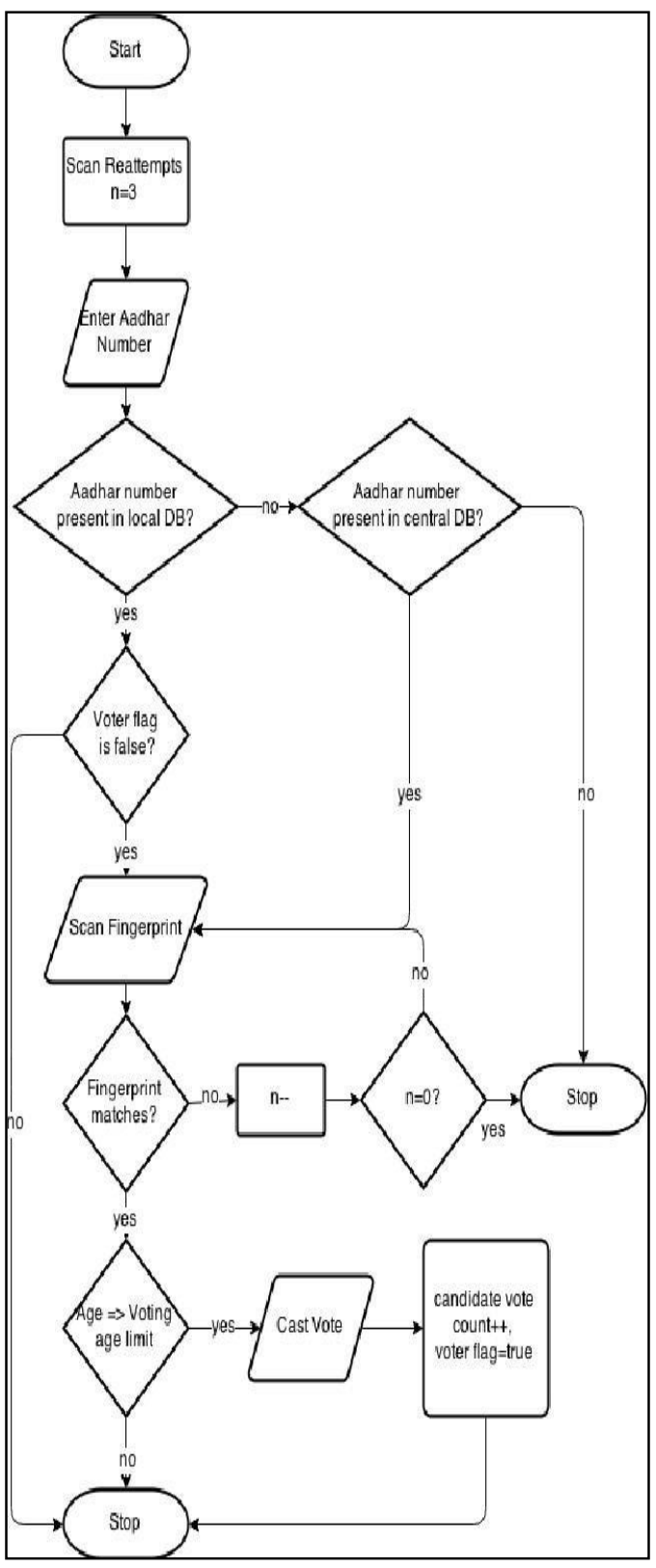

Figure 6. Flowchart of EVM Using Fingerprint Scanner for Better Authentication.

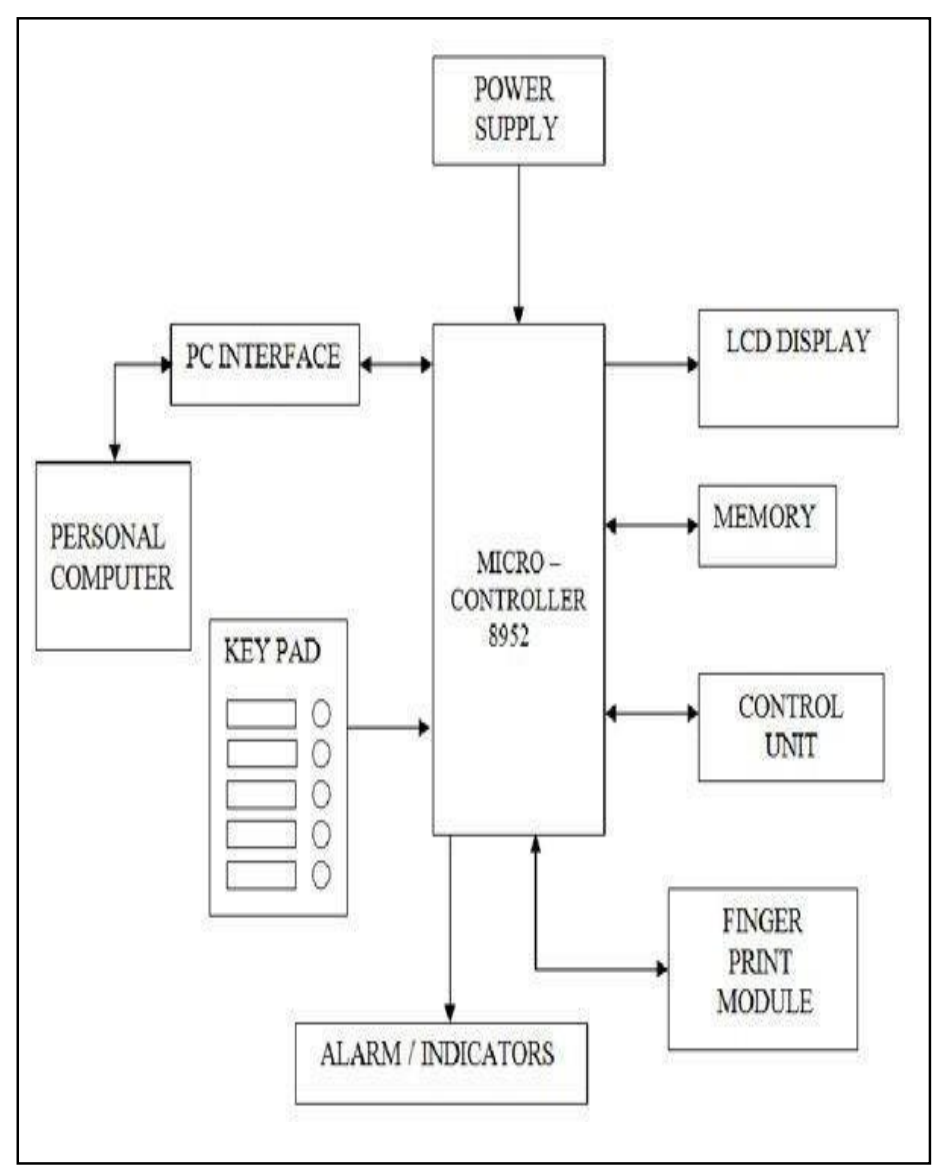

Figure 7. Block Diagram with Fingerprint Scanner

\section{CONCLUSION}

The architecture and operation of a microcontroller-based Electronic Voting Machine have been outlined in this paper. This project is economical, and less manpower is required. Its compact size saves transportation cost and produces results in no time. EVMs have been implemented successfully with full legal support. They are fast and help in fair conduction of elections.

\section{ACKNOWLEDGMENT}

The authors gratefully acknowledge the contributions of Alfred J. Gillespie for the invention of EVMs and the Election Commission of India in alliance with Bharat Electronics Limited (BEL)-and Electronics Corporation of India Limited (ECIL)-for the origination of EVMs in India. Also, the authors would like to thank their mentor Professor Narendra Kumar for his constant guidance which made the completion of this paper possible. 


\section{REFERENCES}

[1] Kraft, S., \& Wall, E. (1986). Experimental microprocessor-based adaptive control system. IEEE Control Systems Magazine, 6(1), 35-40.

[2]. Yung, R., Rusu, S., \& Shoemaker, K. (2002, September). Future trend of microprocessor design. In Proceedings of the 28th European Solid-State Circuits Conference (pp. 43-46). IEEE..

[3]. Weaver, A. C. (1978, January). A real-time, multi-task programming language for microprocessor-based industrial process control. In Proceedings of the 1978 annual conference-Volume 2 (pp. 522-525).

[4]. Chen, C. J., Cheng, W. M., Tsai, H. Y., \& Wu, J. C. (2009). A quasi-delay-insensitive microprocessor core Implementation for Microcontrollers. Journal of information science and engineering, 25(2), 543-557.

[5]. Lee, J. H., Lee, W. C., \& Cho, K. R. (2002, August). A novel asynchronous pipeline architecture for CISC type embedded controller, A8051. In The 2002 45th Midwest Symposium on Circuits and Systems, 2002. MWSCAS-2002. (Vol. 2, pp. II-II). IEEE.

[6] Staunstrup, J., \& Wolf, W. (2013). Hardware/software co-design: principles and practice. Springer Science \& Business Media.

[6] Mazidi, M. A., Mazidi, J. G., \& McKinlay, R. D. (2005). The 8051 microcontroller and embedded systems using assembly and $C$. Dept. of Computer Science and Information Engineering National Cheng Kung University, TAIWAN.

[7] Ali, M. M. (2007). The 8051 Microcontroller and Embedded Systems: Using Assembly and C. Pearson Education India.

[8] Calcutt, D., Cowan, F., \& Parchizadeh, H. (2003). 8051 Microcontroller: An Applications Based Introduction. Elsevier.

[9] Schultz, T. W. (2004). C and the 8051. PageFree Publishing, Inc.. 